\title{
Human rhinovirus infection induces local and systemic immunological tolerance in healthy volunteers
}

RM Koch ${ }^{1 *}$, M Kox $^{1}$, G Ferwerda ${ }^{2}$, J Gerretsen ${ }^{1}$, S ten Bruggencate ${ }^{1}, J G$ van der Hoeven ${ }^{1}$, Ml de Jonge ${ }^{2}$, P Pickkers $^{1}$ From ESICM LIVES 2015

Berlin, Germany. 3-7 October 2015

\section{Introduction}

A large proportion of ICU patients suffer from respiratory virus infections. This is often complicated by secondary infections, suggesting increased vulnerability in these patients. Recent work has shown that in bacterial sepsis, a immunosuppressive state called "immunoparalysis" accounts for this increased vulnerability. However, virus-induced immunoparalysis is largely unstudied.
Human Rhinoviruses (HRVs) are the most frequent cause of the common cold. The "experimental cold model" is widely used to investigate the pathogenesis of HRV infection. However, the effects of repeated HRV exposure and thus possible development of virusinduced immunoparalysis have never been studied. Furthermore, although the virulent HRV-C can cause systemic and severe infections in both children and

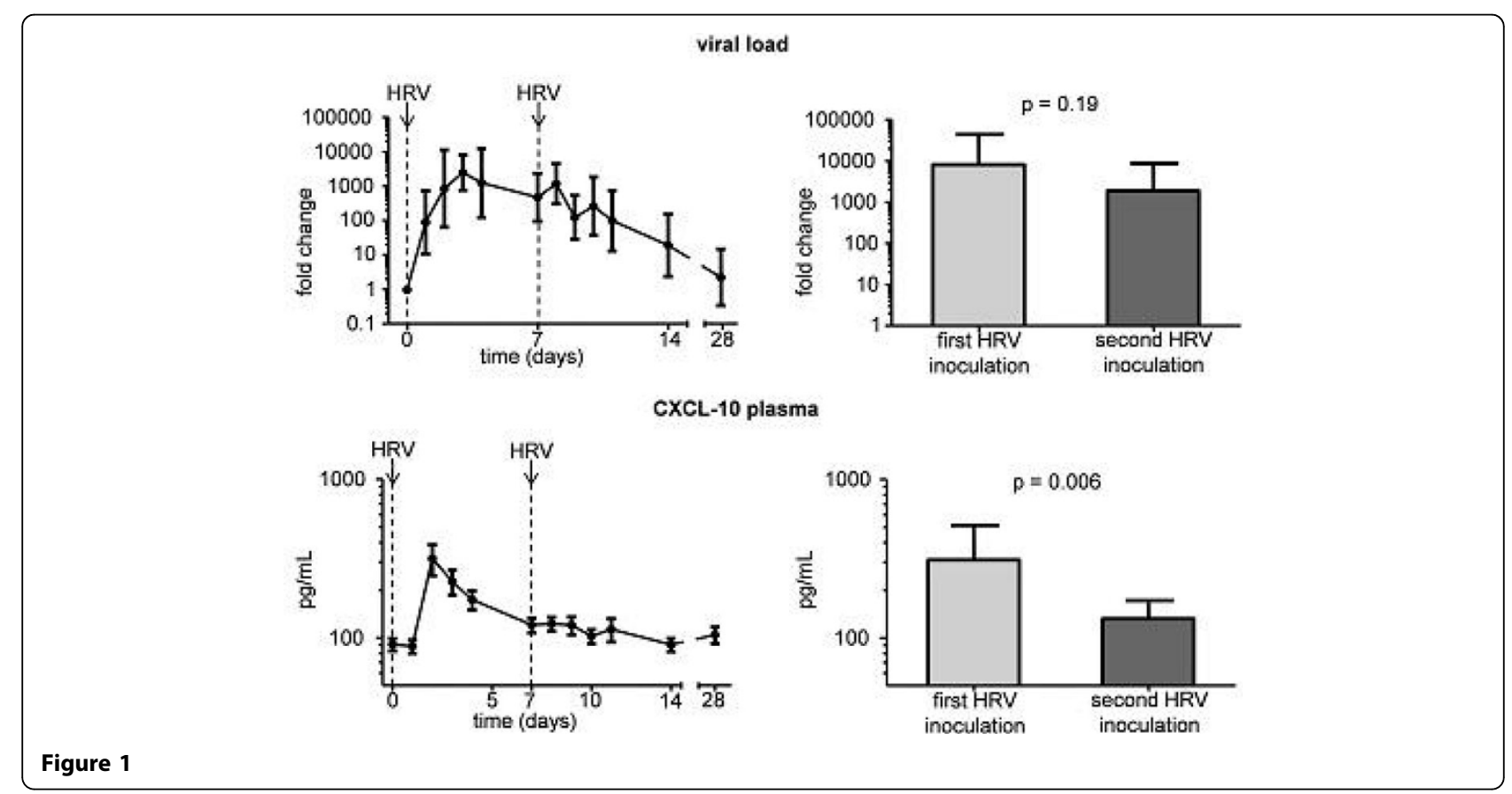

${ }^{1}$ Radboud University Medical Center, Intensive Care, Nijmegen, Netherlands

Full list of author information is available at the end of the article

(c) 2015 Koch et al.; This is an Open Access article distributed under the terms of the Creative Commons Attribution License (http:// creativecommons.org/licenses/by/4.0), which permits unrestricted use, distribution, and reproduction in any medium, provided the original work is properly cited. 


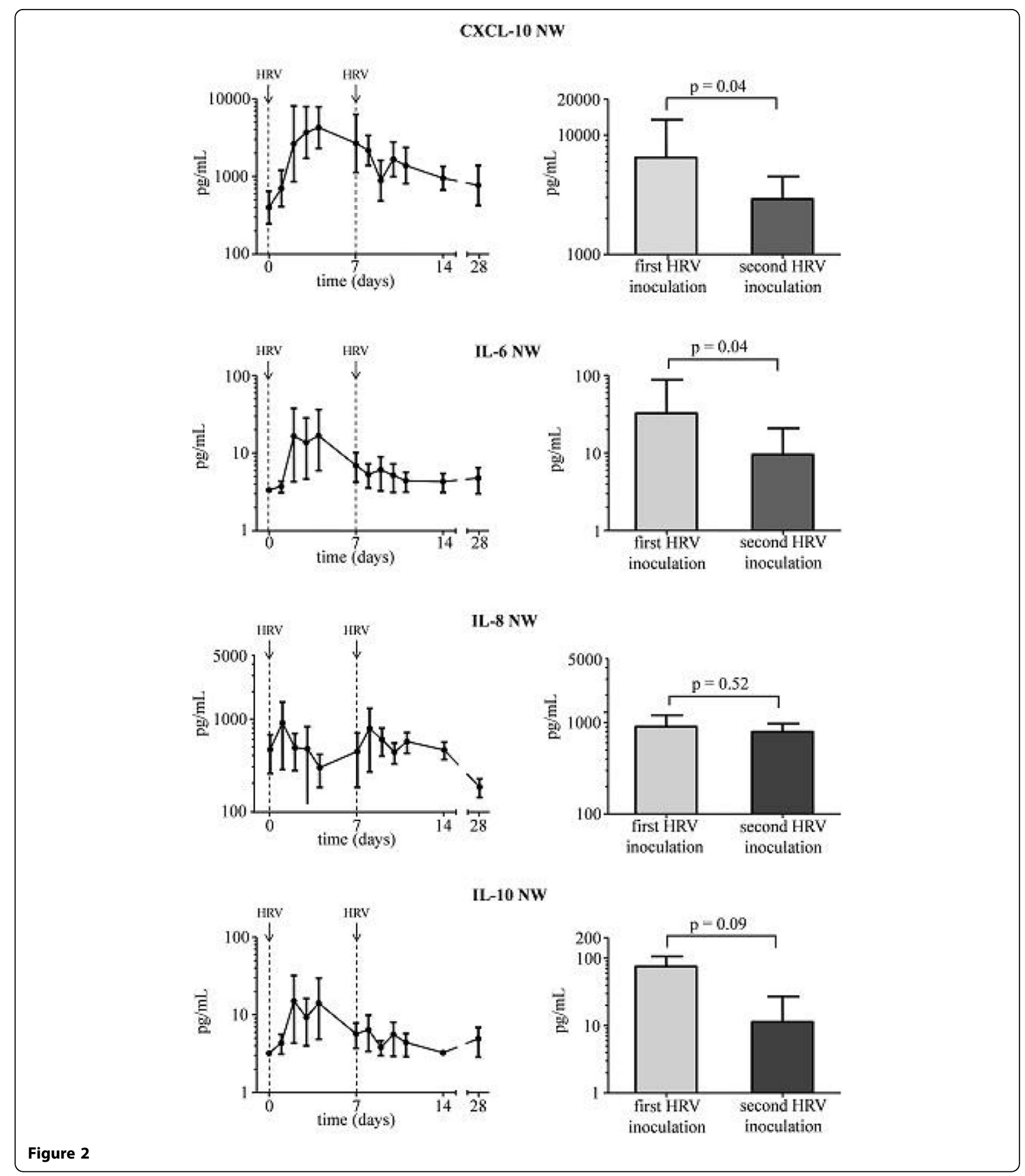

adults and the less virulent HRV-A strain can cause severe infections in immunocompromised patients as well, the HRV-A-induced systemic inflammatory and lower respiratory tract effects have never been studied in healthy subjects. Finally, it remains to be determined, whether serostatus alters the HRV-induced inflammatory response. 


\section{Objectives}

1 . To investigate the effects of serostatus and repeated HRV exposure on the HRV-induced inflammatory response.

2. To assess HRV-induced systemic inflammatory and lower respiratory tract effects.

\section{Methods}

In this randomized, double-blind, placebo-controlled study, 40 healthy, non-smoking, non-asthmatic male and female (1:1) subjects (seronegative: $\mathrm{n}=22$, seropositive: $\mathrm{n}=18)$ were inoculated with HRV-16 (HRV-A) $(\mathrm{n}=20)$ or placebo $(n=20)$. One week later, all subjects were inoculated with HRV-16. Nasal wash, blood samples and peak expiratory flow were obtained daily.

\section{Results}

HRV-inoculation resulted in an infection rate of $82 \%$. In seropositive subjects, HRV infection did not cause a local or systemic inflammatory response. In seronegative subjects, HRV infection resulted in increased levels of CXCL-10 (both in plasma and nasal wash), IL-6, IL-8, and IL-10 (in nasal wash). Despite similar viral load (Figure 1, upper panels), levels of CXCL-10 and IL-6 in nasal wash showed no increase but further declined upon the second HRV inoculation, and a similar trend was observed for IL-10 (Figure 2). Furthermore, the increase in plasma CXCL-10 levels observed after the first HRV inoculation was abolished upon the second HRV inoculation (Figure 1, lower panels). HRV infection did not affect peak expiratory flow.

\section{Conclusions}

HRV infection does not result in an inflammatory response in seropositive subjects and exerts no effects on the lower respiratory tract. Furthermore, a second inoculation with HRV one week after the first results in a diminished local and systemic inflammatory response. This could be an explanation for the increased vulnerability towards secondary infections after respiratory virus infections in ICU patients.

\section{Grant Acknowledgment}

This study was funded by an EFRO grant (2011-013287).

\section{Authors' details}

${ }^{1}$ Radboud University Medical Center, Intensive Care, Nijmegen, Netherlands.

${ }^{2}$ Radboud University Medical Center, Pediatrics, Nijmegen, Netherlands.

Published: 1 October 2015
doi:10.1186/2197-425X-3-S1-A828

Cite this article as: Koch et al:: Human rhinovirus infection induces local and systemic immunological tolerance in healthy volunteers. Intensive Care Medicine Experimental 2015 3(Suppl 1):A828.

\section{Submit your manuscript to a SpringerOpen ${ }^{\mathcal{D}}$ journal and benefit from:}

- Convenient online submission

- Rigorous peer review

- Immediate publication on acceptance

- Open access: articles freely available online

- High visibility within the field

- Retaining the copyright to your article

Submit your next manuscript at $>$ springeropen.com 